\title{
Dynamical Techniques for Analyzing Iterative Schemes with Memory
}

\author{
Neha Choubey $\mathbb{D}^{1},{ }^{1}$ A. Cordero ${ }^{(D},{ }^{2}$ J. P. Jaiswal ${ }^{\circ},{ }^{3}$ and J. R. Torregrosa ${ }^{2}{ }^{2}$ \\ ${ }^{1}$ Department of Mathematics, Oriental Institute of Science and Technology, Bhopal 462021, India \\ ${ }^{2}$ Instituto de Matemática Multidisciplinar, Universitat Politècnica de València, Cno. de Vera s/n, 46022 València, Spain \\ ${ }^{3}$ Department of Mathematics, Maulana Azad National Institute of Technology, Bhopal 462051, India
}

Correspondence should be addressed to J. R. Torregrosa; jrtorre@mat.upv.es

Received 29 January 2018; Accepted 20 May 2018; Published 28 June 2018

Academic Editor: Peter Giesl

Copyright ( $) 2018$ Neha Choubey et al. This is an open access article distributed under the Creative Commons Attribution License, which permits unrestricted use, distribution, and reproduction in any medium, provided the original work is properly cited.

We construct a new biparametric three-point method with memory to highly improve the computational efficiency of its original partner, without adding functional evaluations. In this way, through different estimations of self-accelerating parameters, we have modified an existing seventh-order method. The parameters have been defined by Hermite interpolating polynomial that allows the accelerating effect. In particular, the $R$-order of the proposed iterative method with memory is increased from seven to ten. A real multidimensional analysis of the stability of this method with memory is made, in order to study its dependence on the initial estimations. Taking into account that usually iterative methods with memory are more stable than their derivative-free partners and the obtained results in this study, the behavior of this scheme shows to be excellent, but for a small domain. Numerical examples and comparison are also provided, confirming the theoretical results.

\section{Introduction}

In recent times, solving nonlinear problems described by $f(x)=0$ is burning difficulty in real-world phenomena. In this direction, numerous iterative methods have been projected (see, e.g., [1-7]). These iterative methods have a noteworthy area of research in numerical analysis, as they can be applied in several areas of pure or applied sciences. Out of them, the most eminent one-point iterative method without memory is the Newton-Raphson scheme, which is given by

$$
x_{n+1}=x_{n}-\frac{f\left(x_{n}\right)}{f^{\prime}\left(x_{n}\right)}, \quad n=0,1, \ldots
$$

and has second-order convergence. One drawback of this method is the clause $f^{\prime}\left(x_{n}\right) \neq 0$, which confines its applications.
The first objective and inspiration to design iterative methods for solving this kind of problem are to get the highest order of convergence with the least computational cost. Therefore, a lot of researchers have paid much interest to construct optimal multipoint methods without memory, in the sense of Kung-Traub's conjecture [8], using $n+1$ functional evaluation which can reach the optimal $2^{n}$. The paper follows three main goals: primarily, to avoid the restriction $f^{\prime}(x) \neq 0$ in the practice; along with the subsequent goal, to develop the proposed families to methods with memory in an approach that achieves convergence $R$-order 10 without any new functional evaluation; and to analyze the dependence of the resulting scheme from the set of initial estimations used.

Multipoint schemes have enormous practical meaning, as they conquer theoretical limits of any point-to-point scheme 
about order and efficiency. They also generate approximations of higher precision and highly improved computer arithmetics, and symbolic calculation has allowed efficient execution of multipoint methods.

On the other hand, iterative schemes with memory utilize information from the recent and preceding iterations. Traub, in [9], designed the first method with memory by a small change in the well-known Steffensen's scheme as follows:

$$
\begin{array}{cc}
u_{n}=x_{n}+\rho_{n} f\left(x_{n}\right), \quad \rho_{n} \neq 0, \\
x_{n+1}=x_{n}-\frac{f\left(x_{n}\right)}{f\left[x_{n}, u_{n}\right]}, \quad n \geq 0,
\end{array}
$$

where $x_{0}$ and $\rho_{0}$ are given; $\rho_{n}$ is a self-accelerating parameter defined as

$$
\rho_{n+1}=\frac{-1}{N_{1}{ }^{\prime}\left(x_{n}\right)}, \quad N_{1}(x)=f\left(x_{n}\right)+\left(x-x_{n}\right) f\left[x_{n}, u_{n}\right], n \geq 0,
$$

where $N_{1}(x)$ is Newton's interpolating polynomial of the first degree. The order of convergence of (2) was 2.41.

More recently, some authors have constructed iterative schemes with memory from optimal procedures of different orders, mainly four (see, e.g., [10-12]), eight ([13-15], among others), or even general $n$-point schemes $[16,17]$. Some good reviews regarding the acceleration of convergence order by using memory are $[18,19]$.

The convergence order of a new method is improved regarding its without memory partner; it is derived by adding self-accelerating parameters but holding the derivatives in the iterative expression. The accelerated convergence rate has been obtained without additional functional evaluations, which results in higher computational efficiency. However, another important aspect of an iterative method to be considered is the numerical stability, that is, the analysis that tells us how dependent the scheme of the initial guesses used is. The dynamical performance of the rational functions associated to iterative schemes is a very useful element to study their dependence on initial estimations. In recent years, complex discrete dynamics has been widely used on iterative methods without memory (see, e.g., [20-23]). Nevertheless, it is known that iterative schemes with memory cannot be analyzed by means of these techniques. This is the reason why the authors focused in [24-26] their qualitative study by transforming them into multidimensional dynamical systems, as their qualitative properties can be analyzed by using standard tools as the classical Hénon map (see [27, 28]).

In this manuscript, we have obtained a multipoint method with memory, to solve the nonlinear equations followed by its convergence and stability analysis. Sections 2, 3, and 4 can be summarized as follows: in Section 2, we design a biparametric three-point iterative scheme with memory. The method has been obtained by employing two self-accelerating parameters, which use current and previous data from available information. These parameters are recalculated at each iteration by using Hermite interpolating polynomials, increasing from 7 to 10 the order of convergence. Section 3 is related to the qualitative analysis of the proposed scheme in terms of the real discrete dynamical system involved. After introducing some basic concepts, the stability of the method on a low degree generic polynomial is studied. In general, it presents a very stable behavior, but also a chaotic one for the values of the parameter in a small domain. In Section 4, some numerical examples are presented confirming the results proven in Section 2.

\section{Convergence Analysis}

In this section, we demonstrate the improvement of the convergence order of the method given by Khattri and Argyros [29] by adding up two different parameters in the first and the third steps. Firstly, we consider the seventh order without memory scheme presented in [29].

$$
\begin{aligned}
y_{n} & =x_{n}-\frac{f\left(x_{n}\right)}{f^{\prime}\left(x_{n}\right)}, \\
z_{n} & =y_{n}-\left(\frac{f\left(y_{n}\right)}{-f^{\prime}\left(x_{n}\right)+2\left(\left(f\left(y_{n}\right)-f\left(x_{n}\right)\right) /\left(y_{n}-x_{n}\right)\right)}\right), \\
x_{n+1} & =z_{n}-\frac{f\left(z_{n}\right)}{\alpha_{1}\left(\left(f\left(y_{n}\right)-f\left(x_{n}\right)\right) /\left(y_{n}-x_{n}\right)\right)+\alpha_{2}\left(\left(f\left(z_{n}\right)-f\left(y_{n}\right)\right) /\left(z_{n}-y_{n}\right)\right)+\left(1-\alpha_{1}-\alpha_{2}\right)\left(\left(f\left(z_{n}\right)-f\left(x_{n}\right)\right) /\left(z_{n}-x_{n}\right)\right)},
\end{aligned}
$$

where $\alpha_{1}=-1$ and $\alpha_{2}=1$. The error equation for the last step of (4) is

$$
e_{n+1}=\left(c_{3}-c_{2}^{2}\right) c_{2}^{2} c_{3} e_{n}^{7}+O\left(e_{n}^{8}\right),
$$

where $c_{j}=f^{(j)}(\alpha) / j ! f^{\prime}(\alpha)$, for $j=2,3, \ldots, n$.
By using two parameters $\gamma$ and $\lambda$ in (4) and replacing $\alpha_{1}=-1$ and $\alpha_{2}=1$, we obtain the biparametric family. 


$$
\begin{gathered}
z_{n}=y_{n}-\left(\frac{f\left(y_{n}\right)}{-f^{\prime}\left(x_{n}\right)+2\left(\left(f\left(y_{n}\right)-f\left(x_{n}\right)\right) /\left(y_{n}-x_{n}\right)\right)}\right), \\
x_{n+1}=z_{n}-f\left(z_{n}\right)\left[-\frac{f\left(y_{n}\right)-f\left(x_{n}\right)}{y_{n}-x_{n}}+\frac{f\left(z_{n}\right)-f\left(y_{n}\right)}{z_{n}-y_{n}}\right. \\
\left.+\frac{f\left(z_{n}\right)-f\left(x_{n}\right)}{z_{n}-x_{n}}+\lambda\left(z_{n}-x_{n}\right)\left(z_{n}-y_{n}\right)\right] .
\end{gathered}
$$

The error equations of each step of (6) are

$$
\begin{aligned}
e_{n, y}= & y_{n}-\alpha=\left(c_{2}-\gamma\right) e_{n}^{2}+\left(-2 c_{2}^{2}-\gamma^{2}+2 c_{2} \gamma+2 c_{3}\right) e_{n}^{3} \\
& +\cdots+O\left(e_{n}^{8}\right), \\
e_{n, z}= & z_{n}-\alpha=\left(\gamma-c_{2}\right)\left(\left(\gamma-c_{2}\right) c_{2}+c_{3}\right) e_{n}^{4} \\
& +\left(8 \gamma c_{2}^{3}-4 c_{2}^{4}+3 \gamma c_{3}-2 c_{3}^{2}+c_{2}^{2}\left(-6 \gamma^{2}+8 c_{3}\right)\right. \\
& \left.+2 c_{2}\left(\gamma^{3}-5 \gamma c_{3}-c_{4}\right)+2 \gamma c_{4}\right) e_{n}^{5}+\cdots+O\left(e_{n}^{8}\right),
\end{aligned}
$$

and

$$
e_{n+1}=\frac{\left(\gamma-c_{2}\right)^{2}\left(\left(\gamma-c_{2}\right) c_{2}+c_{3}\right)\left(-\lambda+f^{\prime}(\alpha) c_{3}\right)}{f^{\prime}(\alpha)} e_{n}^{7}+O\left(e_{n}^{8}\right) .
$$

From (9), we can assure that the order of convergence of (6) is still seven, with the independence of parameters $\gamma$ and $\lambda$. This order can be improved from seven to ten, by taking $\gamma=c_{2}$ and $\lambda=f^{\prime}(\alpha) c_{3}$, but root $\alpha$ is not known.

So, to improve the rate of convergence of (6), we recalculate the value of parameters $\gamma$ and $\lambda$ in each iteration, by taking $\gamma \approx c_{2}$ and $\lambda \approx f^{\prime}(\alpha) c_{3}$, as $f^{\prime}(\alpha), f^{\prime \prime}(\alpha)$, and $f^{\prime \prime \prime}(\alpha)$ are not provided. We denote by $\gamma_{n}$ and $\lambda_{n}$ these estimations, and they are computed by using the current and previous iteration satisfying $\lim _{n \rightarrow \infty} \gamma_{n}=c_{2}=f^{\prime \prime}(\alpha) / 2 f^{\prime}(\alpha)$ and $\lim _{n \rightarrow \infty} \lambda_{n}=f^{\prime}(\alpha) c_{3}=f^{\prime \prime \prime}(\alpha) / 6$. We consider the following formulas for $\gamma_{n}$ and $\lambda_{n}$, using Hermite interpolating polynomials of different degrees:

$$
\begin{aligned}
& \gamma_{n}=\frac{H_{5}^{\prime \prime}\left(x_{n}\right)}{2 f^{\prime}\left(x_{n}\right)}, \\
& \lambda_{n}=\frac{H_{6}^{\prime \prime \prime}\left(y_{n}\right)}{6} .
\end{aligned}
$$

Theorem 1. Let $f: I \subseteq \mathbb{R} \rightarrow \mathbb{R}$ be a sufficiently differentiable function and $H_{m}(x)$ be the interpolating polynomial of Hermite with mth degree that interpolates $f(x)$ at nodes $y_{n}, x_{n}$, $x_{n}, t_{0}, \ldots, t_{m-3}$ belonging to interval $I$, and the derivative $f^{(m+1)}$ is continuous in I and the Hermite interpolating polynomial $H_{m}(x)$ satisfies conditions $H_{m}\left(y_{n}\right)=f\left(y_{n}\right), H_{m}\left(x_{n}\right)=$ $f\left(x_{n}\right), \quad H_{m}^{\prime}\left(x_{n}\right)=f^{\prime}\left(x_{n}\right)$, and $H_{m}\left(t_{j}\right)=f\left(t_{j}\right), j=0,1, \ldots$, $m-3$.

Let us define $e_{t, j}=t_{j}-\alpha$ and $j=0,1, \ldots, m-2$ and

(i) All nodes $y_{n}, x_{n}, t_{0}, \ldots, t_{m-3}$ are close enough to zero $\alpha$. (ii) Conditions $e_{n}=x_{n}-\alpha=O\left(e_{t, 0} \ldots e_{t, m-3}\right)$ and $e_{n, y}=$ $y_{n}-\alpha=O\left(e_{n}^{2}, e_{t, 0} \ldots e_{t, m-3}\right)$ hold. Then,

$$
\lambda_{n}-f^{\prime}(\alpha) c_{3} \sim(-1)^{m-3} f^{\prime}(\alpha) c_{m+1} \prod_{j=0}^{m-3} e_{t, j}
$$

Proof. It is known that the error expression of $H_{m}(x)$ can be obtained by

$f(x)-H_{m}(x)=\frac{f^{(m+1)}(\xi)}{(m+1) !}\left(x-y_{n}\right)\left(x-x_{n}\right)^{2} \prod_{j=0}^{m-3}\left(x-t_{j}\right), \quad \xi \in I$.

After differentiating (12) at $x=y_{n}$, we get

$$
\begin{aligned}
& f^{\prime \prime \prime}\left(y_{n}\right)-H_{m}^{\prime \prime \prime}\left(y_{n}\right) \\
& =6 \frac{f^{(m+1)}(\xi)}{(m+1) !} \\
& {\left[\prod_{j=0}^{m-3}\left(y_{n}-t_{j}\right)+2\left(y_{n}-x_{n}\right)\right.} \\
& \cdot \sum_{k=0}^{m-3}\left(\prod_{j \neq k, j=0}^{m-3}\left(y_{n}-t_{j}\right)\right)+\frac{\left(y_{n}-x_{n}\right)^{2}}{2} \\
& \left.\cdot \sum_{k=0}^{m-3} \sum_{i \neq k, i=0}^{m-3}\left(\prod_{j \neq k, j \neq i, j=0}^{m-3}\left(y_{n}-t_{j}\right)\right)\right], \quad \xi \in I, \\
& H_{m}^{\prime \prime \prime}\left(y_{n}\right)=f^{\prime \prime \prime}\left(y_{n}\right)-6 \frac{f^{(m+1)}(\xi)}{(m+1) !} \\
& {\left[\prod_{j=0}^{m-3}\left(y_{n}-t_{j}\right)+2\left(y_{n}-x_{n}\right) \sum_{k=0}^{m-3}\right.} \\
& \left(\prod_{j \neq k, j=0}^{m-3}\left(y_{n}-t_{j}\right)\right)+\frac{\left(y_{n}-x_{n}\right)^{2}}{2} \sum_{k=0}^{m-3} \sum_{i \neq k, i=0}^{m-3} \\
& \left.\left(\prod_{j \neq k, j \neq i, j=0}^{m-3}\left(y_{n}-t_{j}\right)\right)\right], \quad \xi \in I .
\end{aligned}
$$

Taylor's development of the derivative of $f$ at $x_{n}, y_{n} \in I$ and $\xi \in I$ about $\alpha$ provides

$$
f^{\prime \prime \prime}\left(y_{n}\right)=f^{\prime}(\alpha)\left(6 c_{3}+24 c_{4} e_{n, y}+O\left(e_{n, y}^{2}\right)\right)
$$

and

$$
f^{(m+1)}(\xi)=f^{\prime}(\alpha)\left((m+1) ! c_{m+1}+(m+2) ! c_{m+2} e_{\xi}+O\left(e_{\xi}^{2}\right)\right),
$$

where $e_{\xi}=\xi-\alpha$. Putting (14) and (15) in (13), we obtain 


$$
\begin{aligned}
H_{m}^{\prime \prime \prime}\left(y_{n}\right)= & 6 f^{\prime}(\alpha) \\
& \cdot\left(c_{3}+4 c_{4} e_{n, y}-c_{m+1}\left(\prod_{j=0}^{m-3}\left(y_{n}-t_{j}\right)+2\left(y_{n}-x_{n}\right)\right.\right. \\
& \cdot \sum_{k=0}^{m-3}\left(\prod_{j \neq k, j=0}^{m-3}\left(y_{n}-t_{j}\right)\right)+\frac{\left(y_{n}-x_{n}\right)^{2}}{2} \sum_{k=0}^{m-3} \sum_{i \neq k, i=0}^{m-3} \\
& \left.\cdot\left(\prod_{j \neq k, j \neq i, j=0}^{m-3}\left(y_{n}-t_{j}\right)\right)\right),
\end{aligned}
$$

which implies

$$
\begin{aligned}
\lambda_{n}= & \frac{H_{m}^{\prime \prime \prime}\left(y_{n}\right)}{6} \sim f^{\prime}(\alpha) \\
& \cdot\left(c_{3}+4 c_{4} e_{n, y}-c_{m+1}\left(\prod_{j=0}^{m-3}\left(y_{n}-t_{j}\right)+2\left(y_{n}-x_{n}\right) \sum_{k=0}^{m-3}\right.\right. \\
& \cdot\left(\prod_{j \neq k, j=0}^{m-3}\left(y_{n}-t_{j}\right)\right)+\frac{\left(y_{n}-x_{n}\right)^{2}}{2} \sum_{k=0}^{m-3} \sum_{i \neq k, i=0}^{m-3} \\
& \left.\cdot\left(\prod_{j \neq k, j \neq i, j=0}^{m-3}\left(y_{n}-t_{j}\right)\right)\right),
\end{aligned}
$$

that is,

$$
\begin{aligned}
\lambda_{n}-f^{\prime}(\alpha) c_{3} \sim f^{\prime}(\alpha) & \left(-c_{m+1}\left(\prod_{j=0}^{m-3}\left(y_{n}-t_{j}\right)+2\left(y_{n}-x_{n}\right) \sum_{k=0}^{m-3}\left(\prod_{j \neq k, j=0}^{m-3}\left(y_{n}-t_{j}\right)\right)\right.\right. \\
+ & \left.\left.\frac{\left(y_{n}-x_{n}\right)^{2}}{2} \sum_{k=0}^{m-3} \sum_{i \neq k, i=0}^{m-3}\left(\prod_{j \neq k, j \neq i, j=0}^{m-3}\left(y_{n}-t_{j}\right)\right)\right)+4 c_{4} e_{n, y}\right)
\end{aligned}
$$

or

$$
\lambda_{n}-f^{\prime}(\alpha) c_{3} \sim(-1)^{m-3} f^{\prime}(\alpha) c_{m+1} \prod_{j=0}^{m-3} e_{t, j}
$$

The concept of the R-order of convergence [30] and the subsequent declaration (see [4], p.287) is used to approximate the order of convergence of (6).

Then, we can prove the following result.

Theorem 2. Let $f: I \subseteq \mathbb{R} \rightarrow \mathbb{R}$ be a sufficiently differentiable function and let $\gamma_{n}$ and $\lambda_{n}$ be the varying parameters in iterative scheme (6) obtained by means of (10). If the initial estimate $x_{0}$ is close enough to a simple root $\alpha$ of $f(x)$, then, the $R$-order of the iterative method is at least 10. We denote this scheme by OM10.

Proof. Let $\left\{x_{n}\right\}$ be the generated sequence by scheme OM10. As it converges to the root $\alpha$ of $f(x)$, with $R$-order $r$, we write

$$
e_{n+1} \sim D_{n, r} e_{n}^{r}
$$

where $e_{n}=x_{n}-\alpha$. If $n \rightarrow \infty$, then, $D_{n, r} \rightarrow D_{r}$. Therefore,

$$
e_{n+1} \sim D_{n, r}\left(D_{n-1, r} e_{n-1}^{r}\right)^{r}=D_{n, r} D_{n-1, r}^{r} e_{n-1}^{r^{2}} .
$$

Let us consider now sequences $\left\{y_{n}\right\}$ and $\left\{z_{n}\right\}$ with $R$-order $p$ and $q$, respectively; then,

$$
e_{n, y} \sim D_{n, p} e_{n}^{p} \sim D_{n, p}\left(D_{n-1, r} e_{n-1}^{r}\right)^{p}=D_{n, p} D_{n-1, r}^{p} e_{n-1}^{r p},
$$

and

$$
e_{n, z} \sim D_{n, q} e_{n}^{q} \sim D_{n, q}\left(D_{n-1, r} e_{n-1}^{r}\right)^{q}=D_{n, q} D_{n-1, r}^{q} e_{n-1}^{r q} .
$$

The following error expression of the method with memory (6) can be obtained by (7), (8), and (9) and the varying parameter $\gamma_{n}$ and $\lambda_{n}$.

$$
\begin{gathered}
e_{\mathrm{n}, y}=y_{n}-\alpha \sim\left(c_{2}-\gamma_{n}\right) e_{n}^{2}, \\
e_{n, z}=z_{n}-\alpha \sim\left(\gamma_{n}-c_{2}\right)\left(\left(\gamma_{n}-c_{2}\right) c_{2}+c_{3}\right) e_{n}^{4},
\end{gathered}
$$

and

$e_{n+1}=x_{n+1}-\alpha \sim \frac{\left(\gamma-c_{2}\right)^{2}\left(\left(\gamma-c_{2}\right) c_{2}+c_{3}\right)\left(-\lambda+f^{\prime}(\alpha) c_{3}\right)}{f^{\prime}(\alpha)} e_{n}^{7}$.

Here, we excluded higher order terms in (24), (25), and (26).

In method OM10, $\gamma_{n}$ is calculated by (10): Hermite interpolating polynomial $H_{5}(x)$ satisfies conditions $H_{5}\left(x_{n}\right)=$ $f\left(x_{n}\right), \quad H_{5}^{\prime}\left(x_{n}\right)=f^{\prime}\left(x_{n}\right), \quad H_{5}\left(z_{n-1}\right)=f\left(z_{n-1}\right), \quad H_{5}\left(y_{n-1}\right)=$ $f\left(y_{n-1}\right), \quad H_{5}\left(x_{n-1}\right)=f\left(x_{n-1}\right), \quad$ and $H_{5}^{\prime}\left(x_{n}-1\right)=f^{\prime}\left(x_{n}-1\right)$. After differentiating twice the error expression of $H_{5}(x)$ at point $x=x_{n}$, we get

$$
\begin{aligned}
H_{5}^{\prime \prime}\left(x_{n}\right)= & f^{\prime \prime}\left(x_{n}\right)-2 \frac{f^{(6)}(\xi)}{6 !}\left({ }_{n}-x_{n-1}\right)^{2} \\
& \cdot\left(x_{n}-z_{n-1}\right)\left(x_{n}-y_{n-1}\right), \quad \xi \in I .
\end{aligned}
$$

By using Taylor's expansion of $f^{\prime}$ and $f^{\prime \prime}$ at $x_{n} \in I$ and $\xi \in I$ about $\alpha$,

$$
\begin{aligned}
& f^{\prime}\left(x_{n}\right)=f^{\prime}(\alpha)\left(1+2 c_{2} e_{n}+3 c_{3} e_{n}^{2}+O\left(e_{n}^{3}\right)\right), \\
& f^{\prime \prime}\left(x_{n}\right)=f^{\prime}(\alpha)\left(2 c_{2}+6 c_{3} e_{n}+O\left(e_{n}^{2}\right)\right),
\end{aligned}
$$

and

$$
f^{(m+1)}(\xi)=f^{\prime}(\alpha)\left((m+1) ! c_{m+1}+(m+2) ! c_{m+2} e_{\xi}+O\left(e_{\xi}^{2}\right)\right) .
$$

From (30), we have

$$
f^{(6)}(\xi)=f^{\prime}(\alpha)\left(6 ! c_{6}+7 ! c_{7} e_{\xi}+O\left(e_{\xi}^{2}\right)\right),
$$

where $e_{\xi}=\xi-\alpha$. By replacing (29) and (31) into (27), we obtain 


$$
H^{\prime \prime}{ }_{5}\left(x_{n}\right) \sim 2 f^{\prime}(\alpha)\left(c_{2}-c_{6} e_{n-1, y} e_{n-1, z} e_{n-1}^{2}+3 c_{3} e_{n}\right) .
$$

Now, dividing (32) by (28),

$$
\gamma_{n}=\frac{H_{5}^{\prime \prime}\left(x_{n}\right)}{2 f^{\prime}\left(x_{n}\right)} \sim\left(c_{2}-c_{6} e_{n-1, y} e_{n-1, z} e_{n-1}^{2}+\left(3 c_{3}-2 c_{2}^{2}\right) e_{n}\right)
$$

or

$$
\gamma_{n}-c_{2} \sim-c_{6} e_{n-1, y} e_{n-1, z} e_{n-1}^{2}
$$

As $\lambda_{n}$ is calculated by (10), the third derivative of Hermite interpolating polynomial $H_{6}(x)$ satisfies

$$
\begin{aligned}
H_{6}^{\prime \prime \prime}\left(y_{n}\right)= & f^{\prime \prime \prime}\left(y_{n}\right)-\frac{f^{(7)}(\xi)}{7 !} \\
& \cdot\left(6\left(y_{n}-x_{n-1}\right)^{2}\left(y_{n}-y_{n-1}\right)\left(y_{n}-z_{n-1}\right)\right. \\
& +16\left(y_{n}-x_{n-1}\right)^{2}\left(y_{n}-x_{n}\right)\left(y_{n}-z_{n-1}\right) \\
& \left.+16\left(y_{n}-x_{n-1}\right)^{2}\left(y_{n}-x_{n}\right)\left(y_{n}-y_{n-1}\right)\right) \\
+ & 16\left(y_{n}-x_{n}\right)^{2}\left(y_{n}-x_{n-1}\left(y_{n}-z_{n-1}\right)\right. \\
+ & 16\left(y_{n}-x_{n}\right)^{2}\left(y_{n}-x_{n-1}\right)\left(y_{n}-y_{n-1}\right) \\
+ & 24\left(y_{n}-x_{n-1}\right)\left(y_{n}-x_{n}\right)\left(y_{n}-y_{n-1}\right)\left(y_{n}-z_{n-1}\right) \\
& +12\left(y_{n}-x_{n-1}\right)^{2}\left(y_{n}-x_{n}\right)^{2}+6\left(y_{n}-x_{n}\right)^{2} \\
& \left.\cdot\left(y_{n}-y_{n-1}\right)\left(y_{n}-z_{n-1}\right)\right), \quad \xi \in I .
\end{aligned}
$$

From (30), we have

$$
f^{(7)}(\xi)=f^{\prime}(\alpha)\left(7 ! c_{7}+8 ! c_{8} e_{\xi}+O\left(e_{\xi}^{2}\right)\right) .
$$

Substituting (14) and (36) into (35), we obtain

$$
H_{6}^{\prime \prime \prime}\left(y_{n}\right) \sim 6 f^{\prime}(\alpha)\left(c_{3}-c_{7} e_{n-1, y} e_{n-1, z} e_{n-1}^{2}\right),
$$

and hence,

$$
\lambda_{n}=\frac{H_{6}^{\prime \prime \prime}\left(y_{n}\right)}{6} \sim f^{\prime}(\alpha)\left(c_{3}-c_{7} e_{n-1, y} e_{n-1, z} e_{n-1}^{2}\right),
$$

or

$$
\lambda_{n}-f^{\prime}(\alpha) c_{3} \sim-f^{\prime}(\alpha) c_{7} e_{n-1, y} e_{n-1, z} e_{n-1}^{2}
$$

According to (24), (25), (26), (34), and (39), we get

$$
\begin{aligned}
e_{n, y} & \sim-c_{6} e_{n-1, y} e_{n-1, z} e_{n-1}^{2}\left(D_{n-1, r} e_{n-1}^{r}\right)^{2} \\
& \sim-c_{6} D_{n-1, p} D_{n-1, q} D_{n-1, r}^{2} e_{n-1}^{p} e_{n-1}^{q} e_{n-1}^{2 r} e_{n-1}^{2} \\
& \sim-c_{6} D_{n-1, p} D_{n-1, q} D_{n-1, r}^{2} e_{n-1}^{p+q+2 r+2}, \\
e_{n, z} & \sim c_{6} e_{n-1, y} e_{n-1, z} e_{n-1}^{2}\left(D_{n-1, r} e_{n-1}^{r}\right)^{4} \\
& \sim c_{6} D_{n-1, p} D_{n-1, q} D_{n-1, r}^{4} e_{n-1}^{p} e_{n-1}^{q} e_{n-1}^{4 r} e_{n-1}^{2} \\
& \sim c_{6} D_{n-1, p} D_{n-1, q} D_{n-1, r}^{4} e_{n-1}^{p+q+4 r+2}
\end{aligned}
$$

and

$$
\begin{aligned}
e_{n+1} \sim & c_{6}^{2}\left(e_{n-1, y} e_{n-1, z} e_{n-1}^{2}\right)^{2}\left(-c_{7} f^{\prime}(\alpha)\left(e_{n-1, y} e_{n-1, z} e_{n-1}^{2}\right)\right) \\
& \cdot\left(D_{n-1, r} e_{n-1}^{r}\right)^{7} \sim-c_{6}^{2} c_{7} f^{\prime}(\alpha) e_{n-1, y}^{3} e_{n-1, z}^{3} e_{n-1}^{6} D_{n-1, r}^{7} e_{n-1}^{7 r} \\
\sim & -f^{\prime}(\alpha) c_{6}^{2} c_{7} D_{n-1, p}^{3} D_{n-1, q}^{3} D_{n-1, r}^{7} e_{n-1}^{3 p} e_{n-1}^{3 q} e_{n-1}^{7 r} e_{n-1}^{6} \\
\sim & -f^{\prime}(\alpha) c_{6}^{2} c_{7} D_{n-1, p}^{3} D_{n-1, q}^{3} D_{n-1, r}^{7} e_{n-1}^{3 p+3 q+7 r+6} .
\end{aligned}
$$

By comparing the exponents of $e_{n-1}$ featuring in the three pairs of relation (22)-(40), (23)-(41), and (21)-(42), the subsequent system is provided.

$$
\begin{array}{r}
2 r+p+q+2=r p, \\
4 r+p+q+2=r q, \\
7 r+3 p+3 q+6=r^{2} .
\end{array}
$$

The unique positive solution of (43) is $r=10, q=5$, and $p=3$. So $R$-order of method (6), for $\gamma_{n}$ and $\lambda_{n}$ being calculated by (10), is at least 10 .

\section{Multidimensional Dynamical Study}

In this section, we build the discrete dynamical system associated to OM10, for overcoming its qualitative analysis. The general iterative expression of a fixed-point procedure that employs two previous iterations in order to get the following one is

$$
x_{n+1}=h\left(x_{n-1}, x_{n}\right), \quad n \geq 1 \text {, }
$$

where $x_{0}$ and $x_{1}$ are initial approximations. But if the scheme is a three-step one, as OM10, not only $x_{n-1}$ but also the subsequent intermediate points $y_{n-1}$ and $z_{n-1}$ must be used to calculate the following iterate $x_{n}$ with a high order of convergence. So the fixed point iteration is expressed as

$$
x_{n+1}=g\left(x_{n-1}, y_{n-1}, z_{n-1}, x_{n}\right), \quad n \geq 1 .
$$

To obtain the fixed points, we define the fixed point multidimensional function $G: \mathbb{R}^{4} \rightarrow \mathbb{R}^{4}$ associated to $g$ by means of

$$
\begin{aligned}
G & \left(x_{n-1}, y_{n-1}, z_{n-1}, x_{n}\right) \\
& =\left(x_{n}, y_{n}, z_{n}, x_{n+1}\right) \\
& =\left(x_{n}, y_{n}, z_{n}, g\left(x_{n-1}, y_{n-1}, z_{n-1}, x_{n}\right)\right), \quad n=1,2, \ldots
\end{aligned}
$$

Therefore, a fixed point of $G$ satisfies $x_{n+1}=x_{n}$ and $x_{n-1}=y_{n-1}=z_{n-1}=x_{n}$.

We define a discrete dynamical system in $\mathbb{R}^{4}$ from function $G: \mathbb{R}^{4} \rightarrow \mathbb{R}^{4}$, given by

$$
G(z, z y, z z, x)=(x, x y, x z, g(z, x y, x z, x)),
$$

where the steps of $n$th and $(n-1)$ th iterations are denoted by $z=x_{n-1}, z y=y_{n-1}, z z=z_{n-1}, x y=y_{n}, x z=z_{n}$, and $x=x_{n}$. Fixed points $(z, z y, z z, x)$ of $G$ satisfy $z=z y=z z=x$ and $x=g(z, x y, x z, x)$. By imposing these conditions to the rational operator $G$, it can be reduced to a real-valued function $\tilde{g}(x)$, to study the asymptotic stability of these points. 
If all the components of a fixed point of $G$ are different from $r, r$ being a zero of the nonlinear scalar function $f$, then, it is called strange fixed point.

Let $G: \mathbb{R}^{4} \rightarrow \mathbb{R}^{4}$ be a vectorial function. The orbit of a point $\bar{x} \in \mathbb{R}^{4}$ is defined as $\left\{\bar{x}, G(\bar{x}), \ldots, G^{n}(\bar{x}), \ldots\right\}$.

A point $x^{*} \in \mathbb{R}^{4}$ is a $k$-periodic point if $G^{k}\left(x^{*}\right)=x^{*}$ and $G^{p}\left(x^{*}\right) \neq x^{*}$, for $p=1,2, \ldots, k-1$.

The stability of fixed points in multivariate operators (see, e.g., [31]) is analyzed as follows.

Theorem 3. Let $G$ from $\mathbb{R}^{m}$ to $\mathbb{R}^{m}$ be of class $\mathscr{C}^{2}$. Assume $x^{*}$ is a $k$-periodic point. Let $\lambda_{1}, \lambda_{2}, \ldots, \lambda_{m}$ be the eigenvalues of $G^{\prime}\left(x^{*}\right)$.

(a) If all the eigenvalues $\lambda_{j}$ have $\left|\lambda_{j}\right|<1$, then, $x^{*}$ is attracting.

(b) If one eigenvalue $\lambda_{j_{0}}$ has $\left|\lambda_{j_{0}}\right|>1$, then, $x^{*}$ is unstable, that is, repelling or saddle.

(c) If all the eigenvalues $\lambda_{j}$ have $\left|\lambda_{j}\right|>1$, then, $x^{*}$ is repelling.

In addition, a fixed point is hyperbolic if $\lambda_{j}$ satisfies $\left|\lambda_{j}\right|$ $\neq 1 \forall j$. In particular, if $\lambda_{i}$ exists such that $\left|\lambda_{i}\right|<1$ and $\lambda_{j}$ such that $\left|\lambda_{j}\right|>1$, then, the hyperbolic point is a saddle point.

Let us remark the difference between the stability of a fixed point $x^{*}$ in one-dimensional dynamics and that in multidimensional dynamics: meanwhile, in the scalar case, the stability of the fixed point depends on the value of the derivative operator at the point: $\left(\left|R^{\prime}\left(x^{*}\right)\right|<1\right.$ means that $x^{*}$ is attracting, superattracting if $\left|R^{\prime}\left(x^{*}\right)\right|=0$ and repulsive if $\left|R^{\prime}\left(x^{*}\right)\right|>1, R$ being the rational function associated to the iterative method applied on a polynomial $p(x)$ ); in the multidimensional case, the eigenvalues of the Jacobian matrix associated to the fixed point operator are the elements that determine the character of the fixed points.

Moreover, a point $x$ is called critical if the associated Jacobian matrix $G^{\prime}(x)$ satisfies $\operatorname{det}\left(G^{\prime}(x)\right)=0$ (in the onedimensional case, a critical point is that what makes the derivative fixed point operator vanish). One way to calculate critical points, for iterative methods of convergence order higher than two, is to find those fixed points with null eigenvalues $\lambda_{j}=0, \forall j$. In the case that they are also fixed points, they are called superattracting, as an extension of the scalar case.

So, $x^{*}$ being an attracting fixed point of $G$, its basin of attraction $\mathscr{A}\left(x^{*}\right)$ is defined as

$$
\mathscr{A}\left(x^{*}\right)=\left\{x^{(0)} \in \mathbb{R}^{n}: G^{m}\left(x^{(0)}\right) \rightarrow x^{*}, m \rightarrow \infty\right\} .
$$

A key result from Julia and Fatou [32] proves the relationship between the existence of free critical points (they are called in this way if they are different from the roots of $p(x))$ and the set of initial points that converge to an attracting periodic point: there is at least one critical point associated with each invariant Fatou component. So observing the orbit of the critical points, all the attracting behaviors can be found. This result is valid as for complex as for real iterative functions. The main drawback is that often the analytic expression of the critical points cannot be found in high-order methods, because of the elevated degree of the polynomials involved in the rational function.

The union of all the basins of attraction defines the Fatou set, usually represented as a dynamical plane. It is numerically constructed starting with a mesh of initial guesses, iterating the method on them and assigning different colors depending on the basin they converge to.

3.1. Dynamical Analysis of OM10. Now, we study the behavior of the operator associated to scheme OM10 on quadratic polynomials. In order to get this aim, the asymptotic stability of the fixed points of the associate rational vector applied on $p(x)=x^{2}-c$, with $c \in \mathbb{R}$, must be analyzed. It is clear that, if $c>0, p(x)$ has two simple real roots, it has one double root at $x=0$ if $c=0$ and $p(x)$ does not have real roots if $c<0$.

We need to calculate the fixed points of the associate fixed point operator on method OM10 on $p(x)=x^{2}-c$, $M(z, z y, z z, \mathrm{x}, c)$, that is, a high-degree rational function depending on the parameter of the polynomial $c$. However, it is not viable to analyze directly the fixed points of this operator, as usually indetermination appears when we calculate the fixed points due to cancelations of factors as $(z-x)$, $(z y-x)$, ansd $(z z-x)$ in the denominator of $M(z, z y, z z$, $x, c)$. In order to avoid this and using that all the fixed points have equal components, we force firstly $z y=z z=x$ and, after simplifying, $z=x$. The resulting one-dimensional reduced operator is

$$
\begin{aligned}
& {\left[M(z, z y, z z, x, c)_{\left.\right|_{z y=z z=x}}\right]_{\left.\right|_{z=x}}} \\
& \quad=\tilde{m}(x, c)=\frac{x^{2}(x-1)^{2}+c\left(6 x^{2}-6 x+1\right)+c^{2}}{2(2 x-1)(c+x(x-1))} .
\end{aligned}
$$

Moreover, there are two values of $c$ which reduce the rational function:

(i) If $c=0$, then,

$$
\tilde{m}(x, 0)=\frac{(-1+x) x}{-2+4 x}
$$

(ii) If $c=1 / 4$, then,

$$
\tilde{m}\left(x, \frac{1}{4}\right)=\frac{5-4 x+4 x^{2}}{-8+16 x}
$$

Proposition 1. The number of real fixed points (and their stability) of $\tilde{m}(x, c)$ depends on the value of parameter $c$ :

(a) If $c<0, p(x)$ has no real roots, so fixed points $s_{1}$ $(c)=(2 / 3)-(1 / 3) \sqrt{1-3 c}$ and $s_{2}(c)=(2 / 3)+(1 / 3)$ $\sqrt{1-3 c}$ are strange, being both repulsive. 


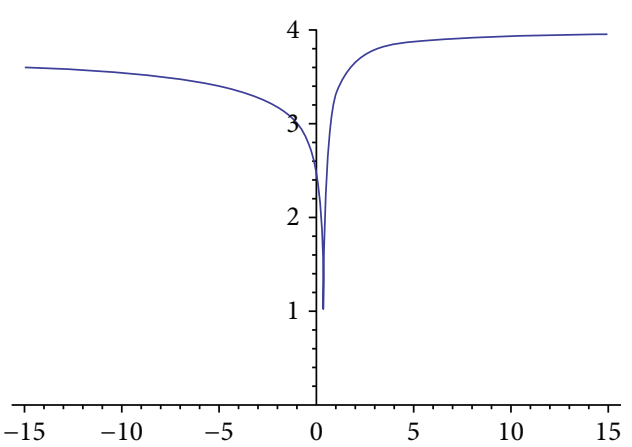

(a) $\left|\lambda_{1}\right|=\left|\tilde{m}\left(s_{1}(c), c\right)\right|$

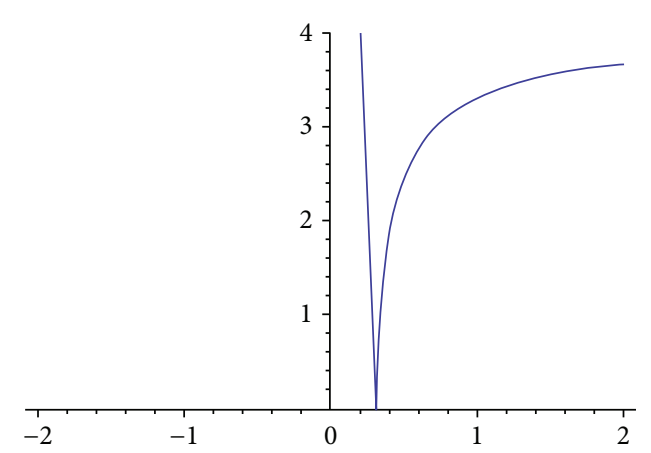

(b) $\left|\lambda_{2}\right|=\left|\tilde{m}\left(s_{2}(c), c\right)\right|$

FIgURE 1: Absolute value of the eigenvalues $\lambda_{i}$ of $M\left(s_{i}(c), s_{i}(c), s_{i}(c), s_{i}(c), c\right), i=1,2$.

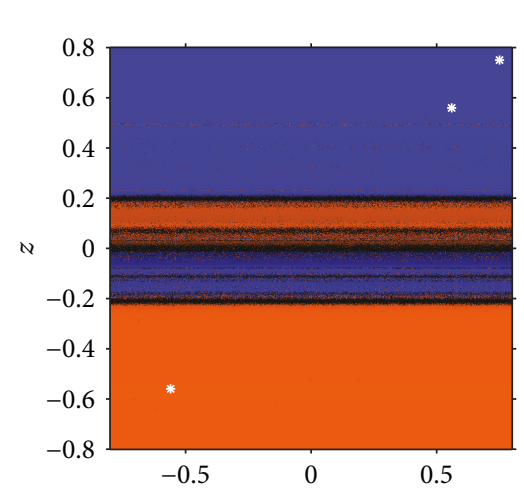

(a) $c=5 / 16$

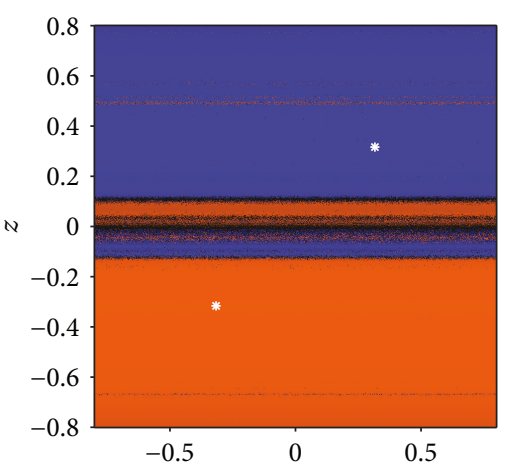

(d) $c=0.1$

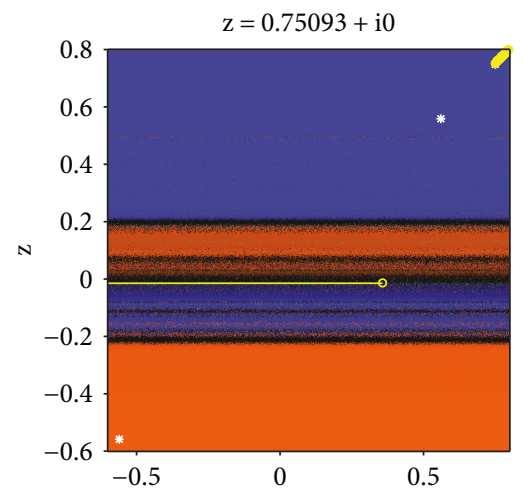

(b) $c=5 / 16$

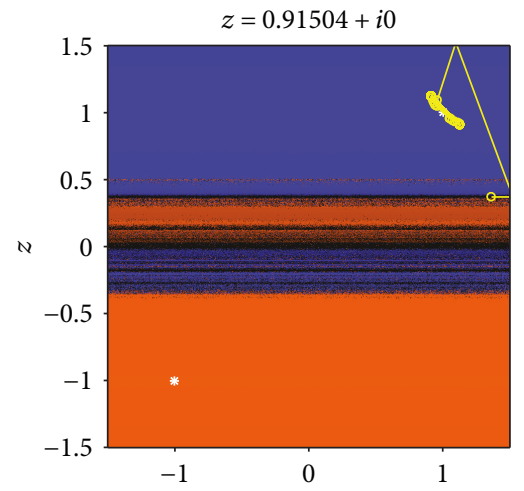

(e) $c=1$

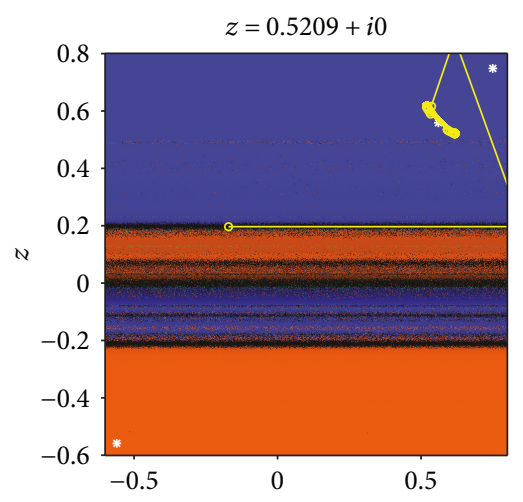

(c) $c=5 / 16$

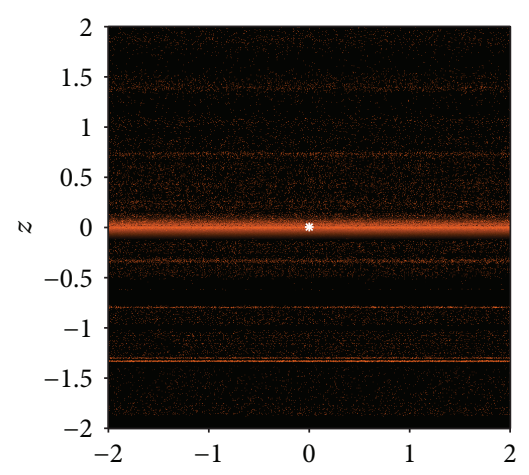

(f) $c=0$

Figure 2: Dynamical planes of OM10 on $p(x)=x^{2}-c$.

(b) If $c=0$, the only (double) root of $p(x), x=0$ is an attracting fixed point of $\tilde{m}(x, 0)$ and also $x=1 / 3$ is a repulsive strange fixed point.

(c) When $0<c<1 / 4$ or $c>1 / 4$, fixed points $(\sqrt{c}, \sqrt{c})$ and $(-\sqrt{c},-\sqrt{c})$ are superattracting (corresponding to the roots of $p(x)$ ). Moreover, $s_{1}(c)$ and $s_{2}(c)$ are strange fixed points if $1 / 4<c \leq 1 / 3 ; s_{1}(c)$ is always repulsive, and $s_{2}(c)$ is attracting if $\left.c \in\right] 7 / 25,1 / 3[$ and superattracting if $c=5 / 16$.

For $c=1 / 4$, there is only one (superattracting) fixed point that is a root of $p(x), x=-1 / 2$ and one strange fixed point, $x=5 / 6$, that is repulsive.
Proof. It is straightforward that identity $\tilde{m}(x, c)=x$ leads us to the fixed points. Specifically,

$$
\begin{aligned}
\tilde{m}(x, c) & =\frac{c^{2}+c\left(6 x^{2}-6 x+1\right)+(x-1)^{2} x^{2}}{2(2 x-1)(c+(x-1) x)} \\
& =x \Leftrightarrow\left(c-x^{2}\right)\left(1+c-4 x+3 x^{2}\right)=0,
\end{aligned}
$$

provided that $(2 x-1)(c+(x-1) x) \neq 0$. That is, the fixed points of $\tilde{m}(x, c)$ are the roots of $p(x)$ or the real roots of polynomial $1+c-4 x+3 x^{2}$. Then, real strange fixed points $s_{1}(c)$ and $s_{2}(c)$ appear when $c \leq 1 / 3$. 


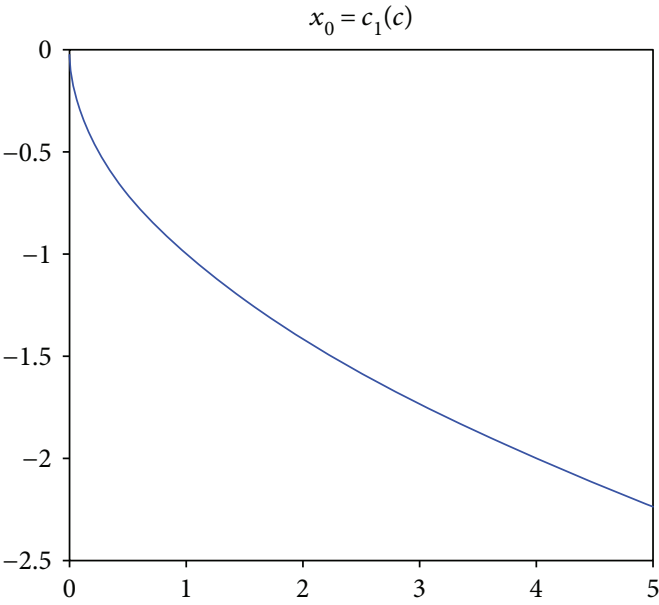

(a) $c_{1}(c)$

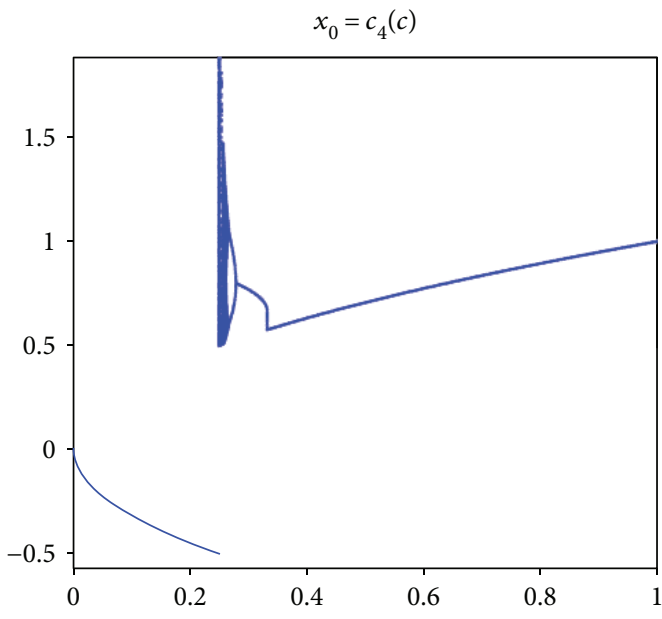

(c) $c_{4}(c)$

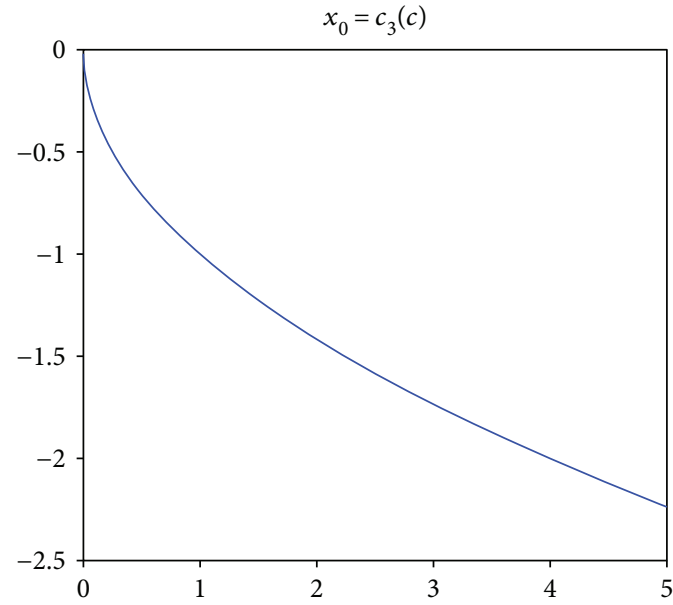

(b) $c_{3}(c)$

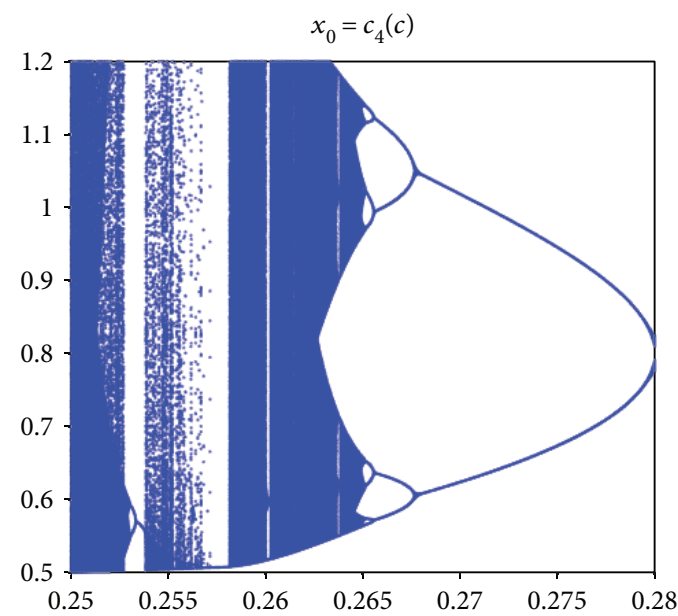

(d) $c_{4}(c)$

FIGURE 3: Bifurcation diagrams of family OM10 on $p(x)$ for free independent critical points.

Regarding their stability, it can be checked that the eigenvalues of the reduced Jacobian matrix coincide with the derivative of $\tilde{m}_{x}(x, c)$ : .

$$
\begin{aligned}
& \operatorname{Eig}\left[M^{\prime}(z, z y, z z, x, c)_{\mid z y=z z=x}\right]_{\mid z=x} \\
& \quad=\tilde{m}_{x}(x, c)=\frac{\left((x-1)^{2}-c\right)\left(x^{2}-c\right)\left(-2 c+2 x^{2}-2 x+1\right)}{2(1-2 x)^{2}(c+(x-1) x)^{2}},
\end{aligned}
$$

and then, their stability is easily stated.

$\left|\tilde{m}_{x}\left(s_{1}(c), c\right)\right|=\left|\frac{-24 c-2 \sqrt{1-3 c}+7}{-6 c+\sqrt{1-3 c}+1}\right|>1, \quad \forall c \in \mathbb{R}$,

and

$\left|\tilde{m}_{x}\left(s_{2}(c), c\right)\right|=\left|\frac{24 c-2 \sqrt{1-3 c}-7}{6 c+\sqrt{1-3 c}-1}\right|<1, \quad$ if $c \in \frac{7}{25}, \frac{1}{3}$, where $\tilde{m}_{x}\left(s_{2}(c), c\right)=0$ for $c=5 / 16$. This is graphically checked in Figure 1.

An example of the behavior stated at Proposition 1 is presented at Figure 2, where the basins of attraction for different values of $c$ are showed. These pictures have been generated by means of a real mesh $(x, z)$ of $800 \times 800$ points, following the routines appearing in [33]. For each initial pair $(x, z)$, intermediate values $z y$ and $z z$ have been generated from $z$ by adding a small random value (in MATLAB code, $z y=$ $z+0.01 *$ rand $(1)$ and $z z=z+0.01 *$ rand $(1))$. When the method is executed on each initial estimation $(x, z)$ under these conditions, the point of the mesh is represented in orange or blue color if the process has converged to one of the roots $\sqrt{c}$ or $-\sqrt{c}$ at a distance lower than $10^{-3}$; other colors correspond to convergence to strange fixed points, and black points to initial guesses that make the method diverge or do not converge after a maximum number of iterations, 200.

Let us remark that $c=5 / 16$ is the value of parameter $c$ that makes the strange fixed point $s_{2}(c)$ superattractor; even in this situation, it is difficult to find initial estimations converging to it (a converging orbit to $s_{2}(5 / 16)$ is plotted in 


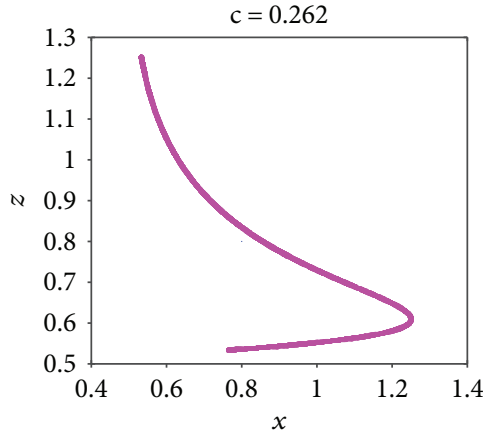

(a) $c=0.262$

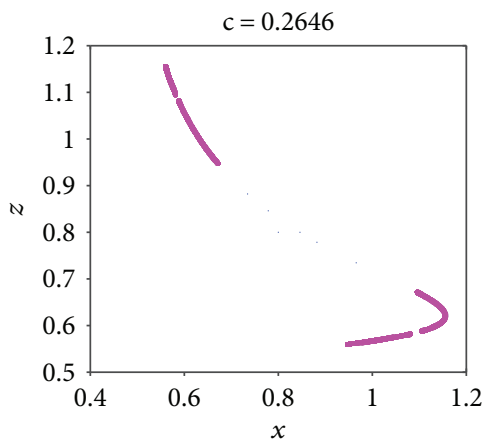

(d) $c=0.2646$

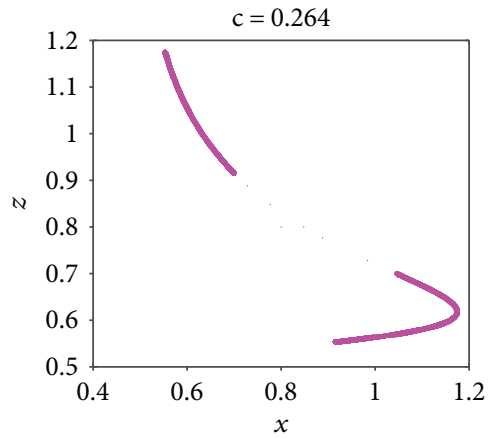

(b) $c=0.264$

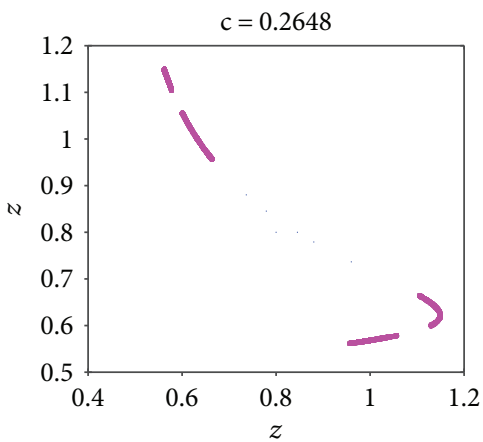

(e) $c=0.2648$

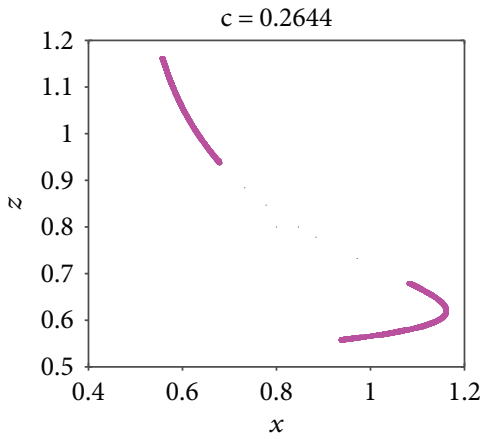

(c) $c=0.2644$

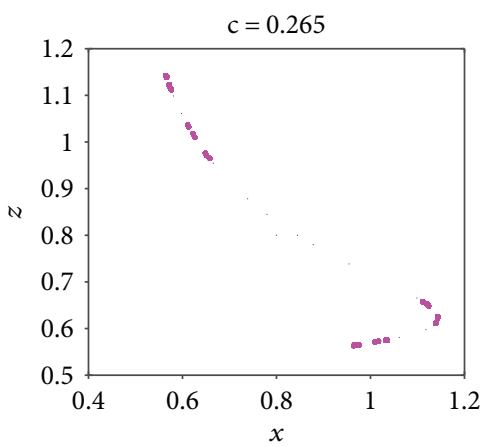

(f) $c=0.265$

Figure 4: Bifurcation diagrams of family OM10 on $p(x)$ for free independent critical points.

TABLE 1: Nonlinear test functions along with their zeros.

\begin{tabular}{lc}
\hline Nonlinear function & Root \\
\hline$f_{1}=x^{3}-x^{2}-1$ & $1.4655 \ldots$ \\
$f_{2}=(x-1)^{3}-1$ & $2.0000 \ldots$ \\
$f_{3}=x^{3}-10$ & $2.1544 \ldots$ \\
\hline
\end{tabular}

Figure 2(b)), as the most of them converge to the roots (orange and blue areas of Figure 2(a)) but some of them converge to 2-periodic orbits (black area appearing in Figure 2(c) where the initial estimation of the orbit is selected). Also, $c=0.1$ is used and convergence is observed (Figure $2(\mathrm{~d})$ ) to both roots with black areas of slow convergence. No other attractors appear. In Figure 2(e), the most frequent behavior is the convergence to the roots, but a black line of convergence to a 2-periodic orbit near $(1,1)$ also appears. In the case of double root, corresponding to $c=0$ (see Figure 2(f)), convergence to $x=z=0$ is very slow.

On the other hand, critical points play a key role in the stability of the system, as they appear always in any basin of attraction. Then, it is necessary, not only to calculate them but also to analyze their asymptotic behavior, in order to detect all the attracting elements: attracting fixed points, periodic orbits, strange attractors, and so on. This is the reason why we calculate the free critical points of $\tilde{m}_{x}(x, c)$, with equal components, in the following result. Maybe other critical points of the multivariate rational functions exist, but their calculation is not feasible due to the high degree of the polynomials (of several variables) involved.
Proposition 2. The number of real critical points of $\tilde{m}(x, c)$ depends on parameter $c, \pm \sqrt{c}, c>0$ are always critical points, and also,

(a) If $c>0, c_{1}(c)=1-\sqrt{c}$ and $c_{2}(c)=1+\sqrt{c}$ are free critical points.

(b) If $c>1 / 4$, there also exist two more free critical points: $c_{3}(c)=(1 / 2)-(1 / 2) \sqrt{-1+4 c}$ and $c_{4}(c)=(1 / 2)+(1 /$ 2) $\sqrt{-1+4 c}$.

The proof of this result is direct from solving $\tilde{m}_{x}(x, c)=$ $\left(\left((x-1)^{2}-c\right)\left(x^{2}-c\right)\left(-2 c+2 x^{2}-2 x+1\right)\right) /\left(2(1-2 x)^{2}\right.$ $\left.(c+(x-1) x)^{2}\right)=0$ or, equivalently, forcing the eigenvalues of the reduced Jacobian matrix to be zero.

Let us remark that, although the dynamics of the method seems to be stable from the dynamical planes plotted and the analysis of the strange fixed points, the presence of any of these critical points can make them bifurcate into periodic orbits or to generate chaos for specific values of $c$. In what follows, we analyze how it changes depending on $c$ and if there exists any other kind of bifurcations that leads to repulsive or attractive points or to periodic or strange attractors.

3.2. Feigenbaum Diagrams. In order to analyze bifurcations, we employ Feigenbaum diagrams of the multidimensional rational function related to OM10 on $p(x)$, by using as initial estimation real critical points described in Proposition 2 and searching those ranges where changes of behavior happen. The critical points are not independent but conjugated two- 


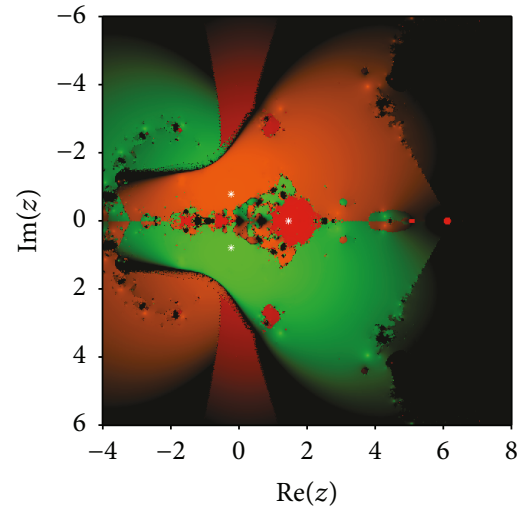

(a) $f_{1}(x)$

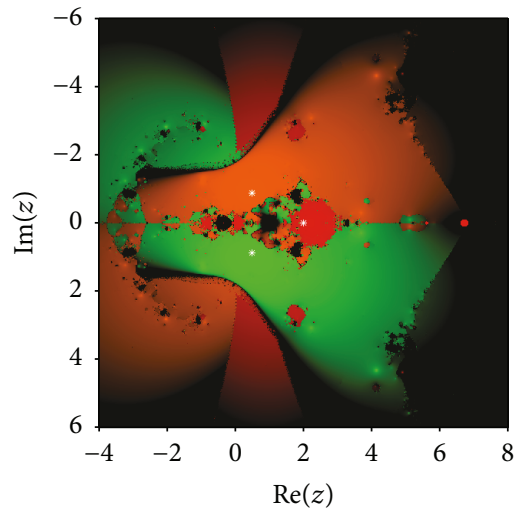

(b) $f_{2}(x)$

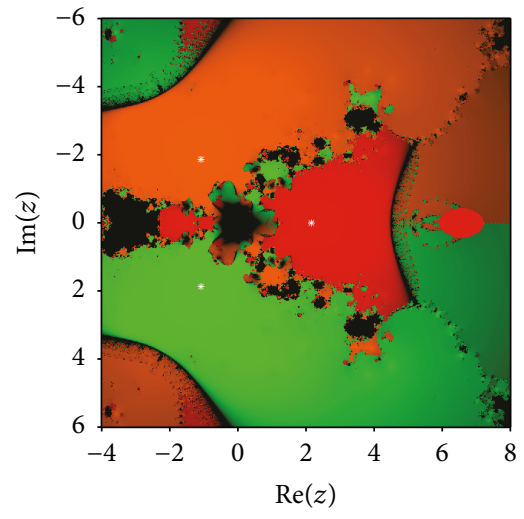

(c) $f_{3}(x)$

Figure 5: Dynamical planes of OM10 on test functions.

by-two: $c_{1}(c)=-1 / c_{2}(c)$ and $c_{3}(c)=-1 / c_{4}(c)$. Nevertheless, it is necessary to analyze the bifurcation diagram corresponding each one of them, as their performance is different.

By using the critical point $c_{1}(c)$ as the starting guess, a bifurcation diagram is observed in Figure 3(a). In this case, it is observed that the critical point is in the basin of attraction of the roots $-\sqrt{c}$, as converges to it for $c>0$. A symmetric behavior is obtained for $c_{2}(c)$ as the initial estimation, belonging to the basin of attraction of the root $\sqrt{c}$. In Figure 3(b), critical point $c_{3}(c)$ shows also convergence to one of the roots, but $c_{4}(c)$ shows a chaotic behavior for $1 / 4<c<1 / 3$, with period-doubling bifurcation cascades as can be observed in Figures 3(c) and 3(d).

We plot the iteration of $\tilde{m}(x, c)$ for the values of $c$ in one of the blue regions of Figure $3(\mathrm{~d})$, in the $(x, z)$ space. In the sequence shown in Figure 4, several strange attractors appear, separated or unified depending on very close values of $c$. These plots have been generated by fixing a value of $c$, with an initial estimation at $x=0.8$. OM10 which has been applied on it, plotting one point per iteration (blue for the first 2000 iterations, green for iteration 2001 to 4000, and magenta for iteration 4001 to 10,000). The resulting images show that several attracting points joint into wandering areas that are unified and separated depending on $c$.

\section{Numerical Comparison}

In this section, after a review of two and three steps with memory methods, we have tried some schemes to be compared with proposed biparametric three-step method OM10, (6). This method is compared with methods XW41 (16-18), XW42 (16-19), XW43 (16-20), XW44 (17-18), XW45 (17-19), and XW46 (17-20) presented in [5], XW5(18-24) given in [1], method SK7 in [29], and methods XW81 (37-34), XW82 (37-35), XW83 (37-36), XW84 (38$34)$, XW85 (38-35), and XW86 (38-36) given in [6] by using the test functions shown in Table 1.

A numerical test of methods with memory is usually made by using starting point $x_{0}$ and also starting values of the accelerating parameters: $\gamma_{0}$ to calculate $y_{1}$ and $z_{1}$ and thereafter $\lambda_{0}$ in order to estimate $x_{1}$. We check the performance of the methods by using this strategy, in order to see if the unstable behavior is avoided or, on the contrary, increased. For this, we use $\gamma_{0}=0.01$ and $\lambda_{0}=2$. It can be observed in Figure 5 that the stability of the method is highly improved when appropriate initial values are considered, not only $x_{0}$ but also the steps $y_{1}$ and $z_{1}$ and, subsequently, the second iterate $x_{1}$. The areas of convergence around the searched roots are wide in spite of the complexity of the nonlinear functions involved, and the behavior of the method is stable.

Table 1 is furnished with the considered nonlinear test functions with their root $(\alpha)$. In the same table, there are infinite numbers of digits after the decimal but we have mentioned only four digits (the nonlinear functions are taken from [6, 7]). In Table 2, the absolute errors $\left|x_{k}-\alpha\right|$ are given for presented method OM10. The computational order of convergence COC approximated using expression (see [34])

$$
C O C \approx \frac{\ln \left|f\left(x_{n+1}\right) / f\left(x_{n}\right)\right|}{\ln \left|f\left(x_{n}\right) / f\left(x_{n-1}\right)\right|},
$$

to check the theoretical order. All the numerical results revealed in Table 2 agree with previous results; for this, we have considered up to 2000 significant digits by using "Set Accuracy" command in Mathematica 8. OM10 has been used to solve the nonlinear functions, and the calculated results are compared with the other methods XW41 (16-18), XW42 (16-19), XW43 (16-20), XW44 (17-18), XW45 (17-19), XW46 (17-20),XW5(18-24), SK7, XW81 (37-34), XW82 (37-35), XW83 (37-36), XW84 (38-34), XW85 (38-35), and XW86 (38-36). In Table 2, "NC" means not convergent. From Table 2, it is very easy to identify that the results obtained by the proposed method are quite superior to the other two- and three-step schemes.

\section{Conclusion}

In this work, we have presented a new family of biparametric three-step schemes with memory to solve nonlinear equations. As a result, we have used two self-correcting parameters, designed by Hermite interpolating polynomials in the seventh-order method to achieve higher order convergence without any additional calculation. The $R$-order of OM10 increases up to 10 . The stability analysis of this family has 
TABLE 2: Numerical comparison of bi-parameter with memory method.

\begin{tabular}{|c|c|c|c|c|c|c|c|}
\hline Example & Method & Guess & $\left|x_{1}-\alpha\right|$ & $\left|x_{2}-\alpha\right|$ & $\left|x_{3}-\alpha\right|$ & $\left|x_{4}-\alpha\right|$ & $\mathrm{COC}$ \\
\hline \multirow{15}{*}{$f_{1}$} & XW41 $(16-18), T_{0}=1.5, a=8$ & 1.3 & $\mathrm{NC}$ & - & - & - & - \\
\hline & XW41 (16-19), $T_{0}=1.5, a=8$ & & NC & - & - & - & - \\
\hline & XW41 (16-20), $T_{0}=1.5, a=8$ & & NC & - & - & - & - \\
\hline & XW41 (17-18), $T_{0}=1.5, b=-2$ & & $0.0437 e-0$ & $0.1582 e-6$ & $0.7900 e-33$ & $0.3499 e-148$ & 4.5591 \\
\hline & XW41 (17-19), $T_{0}=1.5, b=-2$ & & $0.0437 e-0$ & $0.4206 e-8$ & $0.1566 e-37$ & $0.1579 e-154$ & 4.3383 \\
\hline & XW41 (17-20), $T_{0}=1.5, b=-2$ & & $0.0437 e-0$ & $0.4206 e-8$ & $0.3135 e-35$ & $0.1015 e-152$ & 4.3310 \\
\hline & XW81 (37-34), $T_{0}=1.5, L=0$ & & $0.0240 e-0$ & $0.2017 e-17$ & $0.3801 e-153$ & $0.1089 e-1374$ & 9.0001 \\
\hline & XW82 (37-35), $T_{0}=1.5, L=0$ & & $0.0240 e-0$ & $0.5752 e-18$ & $0.3290 e-174$ & $0.1235 e-1736$ & 10.000 \\
\hline & XW83 (37-36), $T_{0}=1.5, L=0$ & & $0.0240 e-0$ & $0.5752 e-18$ & $0.3290 e-174$ & $0.1250 e-1736$ & 10.000 \\
\hline & XW84 (38-34), $T_{0}=1.5, K=6$ & & $0.0828 e-0$ & $0.2901 e-10$ & $0.3076 e-80$ & $0.1528 e-647$ & 8.1216 \\
\hline & XW85 (38-35), $T_{0}=1.5, K=6$ & & $0.0528 e-0$ & $0.4136 e-13$ & $0.1322 e-124$ & $0.1472 e-1239$ & 10.000 \\
\hline & XW86 (38-36), $T_{0}=1.5, K=6$ & & $0.0528 e-0$ & $0.4136 e-13$ & $0.1322 e-124$ & $0.1472 e-1239$ & 10.000 \\
\hline & XW5 (18-24), $T_{0}=-1.5, K_{0}=-1.5$ & & $0.8364 e-3$ & $0.1055 e-13$ & $0.7715 e-66$ & $0.1276 e-315$ & 4.7910 \\
\hline & SK7, $\alpha_{1}=-1, \alpha_{2}=1$ & & $0.2002 e-1$ & $0.4807 e-41$ & $0.1535 e-289$ & $0.3491 e-2029$ & 7.0000 \\
\hline & OM10 (4)-(8), $\gamma=1.5, \lambda=1.2$ & & $0.7454 e-9$ & $0.1755 e-72$ & $0.9235 e-729$ & $0.1496 e-7291$ & 10.000 \\
\hline \multirow{15}{*}{$f_{2}$} & XW41 (16-18), $T_{0}=1.5, a=8$ & 2.5 & NC & - & - & - & - \\
\hline & XW41 (16-19), $T_{0}=1.5, a=8$ & & NC & - & - & - & - \\
\hline & XW41 (16-20), $T_{0}=1.5, a=8$ & & $\mathrm{NC}$ & - & - & - & - \\
\hline & XW41 (17-18), $T_{0}=1.5, b=-2$ & & NC & - & - & - & - \\
\hline & XW41 (17-19), $T_{0}=1.5, b=-2$ & & NC & - & - & - & - \\
\hline & XW41 (17-20), $T_{0}=1.5, b=-2$ & & NC & - & - & - & - \\
\hline & XW81 (37-34), $T_{0}=1.5, L=0$ & & $3.2783 e-4$ & $5.6640 e-33$ & $7.5622 e-293$ & $9.9847 e-2632$ & 9.0000 \\
\hline & XW82 (37-35), $T_{0}=1.5, L=0$ & & $3.2783 e-3$ & $1.7686 e-36$ & $3.6959 e-354$ & $5.8712 e-3586$ & 10.000 \\
\hline & XW83 (37-36), $T_{0}=1.5, L=0$ & & $3.2783 e-3$ & $1.7686 e-36$ & $3.6959 e-354$ & $5.8712 e-3586$ & 10.000 \\
\hline & XW84 (38-34), $T_{0}=1.5, K=6$ & & NC & - & - & - & - \\
\hline & XW85 (38-35), $T_{0}=1.5, K=6$ & & NC & - & - & - & - \\
\hline & XW86 (38-36), $T_{0}=1.5, K=6$ & & NC & - & - & - & - \\
\hline & XW5 (18-24), $T_{0}=-1.5, K_{0}=-1.5$ & & $1.2283 e-2$ & $4.9823 e-11$ & $5.6852 e-53$ & $1.0497 e-262$ & 5.000 \\
\hline & $\mathrm{SK} 7, \alpha_{1}=-1, \alpha_{2}=1$ & & $1.0478 e-4$ & $3.0825 e-29$ & $5.8764 e-201$ & $5.3772 e-1403$ & 7.0000 \\
\hline & OM10 (4)-(8), $\gamma=1.5, \lambda=1.2$ & & $1.5515 e-3$ & $3.9470 e-30$ & $4.5314 e-296$ & $1.8024 e-2955$ & 10.000 \\
\hline \multirow{15}{*}{$f_{3}$} & XW41 (16-18), $T_{0}=-1.5, a=8$ & 2.0 & $2.5035 e-3$ & $5.5559 e-15$ & $6.3146 e-68$ & $2.3358 e-309$ & 4.8601 \\
\hline & XW42 (16-19), $T_{0}=-1.5, a=8$ & & $2.5035 e-3$ & $1.0056 e-15$ & $8.3197 e-70$ & $7.0470 e-305$ & 4.3466 \\
\hline & XW43 (16-20), $T_{0}=-1.5, a=8$ & & $2.5035 e-3$ & $1.0056 e-15$ & $1.6639 e-69$ & $4.5101 e-303$ & 4.3429 \\
\hline & XW44 (17-18), $T_{0}=-1.5, b=-2$ & & $1.6966 e-3$ & $1.0769 e-15$ & $3.750 e-71$ & $4.4929 e-324$ & 4.5604 \\
\hline & XW45 (17-19), $T_{0}=-1.5, b=-2$ & & $1.6966 e-3$ & $1.4415 e-16$ & $1.6141 e-73$ & $4.4565 e-321$ & 4.3469 \\
\hline & XW46 (17-20), $T_{0}=-1.5, b=-2$ & & $1.6966 e-3$ & $1.4415 e-16$ & $3.2282 e-73$ & $2.8521 e-319$ & 4.3434 \\
\hline & XW81 (37-34), $T_{0}=-1.5, L=0$ & & $1.4745 e-7$ & $1.2463 e-66$ & $1.9291 e-598$ & $9.8391 e-5285$ & 9.0000 \\
\hline & XW82 (37-35), $T_{0}=-1.5, L=0$ & & $1.4745 e-7$ & $5.9981 e-73$ & $7.4408 e-727$ & $6.4227 e-7266$ & 10.000 \\
\hline & XW83 (37-36), $T_{0}=-1.5, L=0$ & & $1.4745 e-7$ & $5.9981 e-73$ & $7.4408 e-727$ & $6.4227 e-7266$ & 10.000 \\
\hline & XW84 (38-34), $T_{0}=-1.5, K=6$ & & $7.7322 e-5$ & $5.9725 e-39$ & $3.3296 e-315$ & $2.3998 e-2359$ & 8.1235 \\
\hline & XW85 (38-35), $T_{0}=-1.5, K=6$ & & $1.5456 e-4$ & $1.6975 e-41$ & $4.3428 e-411$ & $5.2174 e-4107$ & 10.000 \\
\hline & XW86 (38-36), $T_{0}=-1.5, K=6$ & & $1.5456 e-4$ & $1.6975 e-41$ & $4.3428 e-411$ & $5.2174 e-4107$ & 10.000 \\
\hline & XW5 (18-24), $T_{0}=-1.5, K_{0}=-1.5$ & & $4.6468 e-4$ & $1.8636 e-19$ & $1.9319 e-96$ & $2.3132 e-481$ & 5.000 \\
\hline & SK7, $\alpha_{1}=-1, \alpha_{2}=1$ & & $7.0030 e-9$ & $1.8357 e-60$ & $1.5607 e-421$ & $5.0127 e-2949$ & 7.0000 \\
\hline & OM10 (4)-(8), $\gamma_{0}=-1.5, \lambda_{0}=-1.5$ & & $3.0734 e-7$ & $3.7135 e-70$ & $2.4628 e-699$ & $4.0535 e-6991$ & 10.000 \\
\hline
\end{tabular}


been made by transforming it in a discrete dynamical system and studying the asymptotical behavior of the fixed and critical points. The method has been shown to be very stable except in few cases. These results have been checked by using dynamical planes, and the proposed method has been compared in performance and computational efficiency with a few existing methods by numerical examples. This confirms the validity of theoretical results.

\section{Data Availability}

The data used to support the findings of this study are available from the corresponding author upon request.

\section{Acknowledgments}

This research has been partially supported by grants from Spanish Ministerio de Economía y Competitividad (MTM2014-52016-C2-2-P) and by Generalitat Valenciana (PROMETEO/2016/089).

\section{Conflicts of Interest}

The authors declare that there are no conflicts of interest regarding the publication of this paper.

\section{References}

[1] X. Wang and T. Zhang, "Higher-order newton-type iterative methods with and without memory for solving nonlinear equations," Mathematical Communications, vol. 19, pp. 91109, 2014.

[2] F. Soleymani, "Some optimal iterative methods and their with memory variants," Journal of the Egyptian Mathematical Society, vol. 21, no. 2, pp. 133-141, 2013.

[3] S. Kumar, V. Kanwar, S. K. Tomar, and S. Singh, "Geometrically constructed families of Newton's method for unconstrained optimization and nonlinear equations," International Journal of Mathematics and Mathematical Sciences, vol. 2011, Article ID 972537, 9 pages, 2011.

[4] G. Alefeld and J. Herzberger, Introduction to Interval Computation, Academic Press, New York, NY, USA, 1983.

[5] X. Wang and T. Zhang, "A new family of newton-type iterative methods with and without memory for solving nonlinear equations," Calcolo, vol. 51, no. 1, pp. 1-15, 2014.

[6] X. Wang and T. Zhang, "Some Newton-type iterative methods with and without memory for solving nonlinear equations," International Journal of Computational Methods, vol. 11, no. 5, pp. 1350078-1350020, 2014.

[7] C. Chun and M. Y. Lee, "A new optimal eighth-order family of iterative methods for the solution of nonlinear equations," Applied Mathematics and Computation, vol. 223, pp. 506519, 2013.

[8] H. T. Kung and J. F. Traub, "Optimal order of one-point and multipoint iteration," Journal of the ACM, vol. 21, no. 4, pp. 643-651, 1974.

[9] J. F. Traub, Iterative Methods for Solution of Equations, Prentice Hall, Englewood Cliffs, NJ, USA, 1964.

[10] A. Cordero, T. Lotfi, P. Bakhtiari, and J. R. Torregrosa, "An efficient two-parametric family with memory for nonlinear equations," Numerical Algorithms, vol. 68, no. 2, pp. 323335, 2015.

[11] M. S. Petković, J. Džunić, and L. D. Petković, “A family of two-point methods with memory for solving nonlinear equations," Applicable Analysis and Discrete Mathematics, vol. 5, no. 2, pp. 298-317, 2011.

[12] F. Zafar, N. Yasmin, M. A. Kutbi, and M. Zeshan, "Construction of tri-parametric derivative free fourth order with and without memory iterative method," Journal of Nonlinear Sciences and Applications, vol. 9, no. 4, pp. 1410-1423, 2016.

[13] X. Wang, J. Džunić, and T. Zhang, "On an efficient family of derivative free three-point methods for solving nonlinear equations," Applied Mathematics and Computation, vol. 219, no. 4, pp. 1749-1760, 2012.

[14] T. Lotfi, F. Soleymani, M. Ghorbanzadeh, and P. Assari, "On the construction of some tri-parametric iterative methods with memory," Numerical Algorithms, vol. 70, no. 4, pp. 835-845, 2015.

[15] F. Soleymani, T. Lotfi, E. Tavakoli, and F. Khaksar Haghani, "Several iterative methods with memory using self-accelerators," Applied Mathematics and Computation, vol. 254, pp. 452-458, 2015.

[16] J. Džunić and M. S. Petković, "On generalized biparametric multipoint root finding methods with memory," Computational and Applied Mathematics, vol. 255, pp. 362-375, 2014.

[17] X. Wang and T. Zhang, "Efficient $n$-point iterative methods with memory for solving nonlinear equations," Numerical Algorithms, vol. 70, no. 2, pp. 357-375, 2015.

[18] M. S. Petković, B. Neta, L. D. Petković, and J. Džunić, Multipoint Methods for Solving Nonlinear Equations, Elsevier, Amsterdam, Netherlands, 2013.

[19] M. S. Petković, B. Neta, L. D. Petković, and J. Džunić, "Multipoint methods for solving nonlinear equations: a survey," Applied Mathematics and Computation, vol. 226, pp. 635660, 2014.

[20] S. Amat, S. Busquier, C. Bermúdez, and Á. A. Magreñán, “On the election of the damped parameter of a two-step relaxed Newton-type method," Nonlinear Dynamics, vol. 84, no. 1, pp. 9-18, 2016.

[21] Y. H. Geum, Y. I. Kim, and Á. A. Magreñán, “A biparametric extension of King's fourth-order methods and their dynamics," Applied Mathematics and Computation, vol. 282, pp. 254-275, 2016.

[22] Á. A. Magreñán and I. K. Argyros, "On the local convergence and the dynamics of Chebyshev-Halley methods with six and eight order of convergence," Journal of Computational and Applied Mathematics, vol. 298, pp. 236-251, 2016.

[23] B. Neta, C. Chun, and M. Scott, "Basins of attraction for optimal eighth order methods to find simple roots of nonlinear equations," Applied Mathematics and Computation, vol. 227, pp. 567-592, 2014.

[24] B. Campos, A. Cordero, J. R. Torregrosa, and P. Vindel, “A multidimensional dynamical approach to iterative methods with memory," Applied Mathematics and Computation, vol. 271, pp. 701-715, 2015.

[25] B. Campos, A. Cordero, J. R. Torregrosa, and P. Vindel, "Stability of King's family of iterative methods with memory," Computational and Applied Mathematics, vol. 318, pp. 504-514, 2017.

[26] A. Cordero, F. Soleymani, J. R. Torregrosa, and F. K. Haghani, "A family of Kurchatov-type methods and its stability," 
Applied Mathematics and Computation, vol. 294, pp. 264-279, 2017.

[27] W. F. Hassan Al-Shameri, "Dynamical properties of the Hénon mapping," International Journal of Mathematical Analysis, vol. 6, no. 49, pp. 2419-2430, 2012.

[28] M. Hénon, "A two-dimensional mapping with a strange attractor," Communications in Mathematical Physics, vol. 50, no. 1 , pp. 69-77, 1976.

[29] S. K. Khattri and I. K. Argyros, "How to develop fourth and seventh order iterative methods," Novi Sad Journal of Mathematics, vol. 40, no. 2, pp. 61-67, 2010.

[30] J. M. Ortega and W. C. Rheinbolt, Iterative Solution of Nonlinear Equations in Several Variables, Academic Press, New York, NY, USA, 2000.

[31] R. C. Robinson, An Introduction to Dynamical Systems, Continous and Discrete, American Mathematical Society, Providence, RI, USA, 2012.

[32] P. Blanchard, "Complex analytic dynamics on the Riemann sphere," Bulletin of the American Mathematical Society, vol. 11, no. 1, pp. 85-142, 1984.

[33] F. I. Chicharro, A. Cordero, and J. R. Torregrosa, "Drawing dynamical and parameters planes of iterative families and methods," The Scientific World Journal, vol. 2013, Article ID 780153, 11 pages, 2013.

[34] L. O. Jay, "A note on Q-order of convergence," BIT Numerical Mathematics, vol. 41, no. 2, pp. 422-429, 2001. 


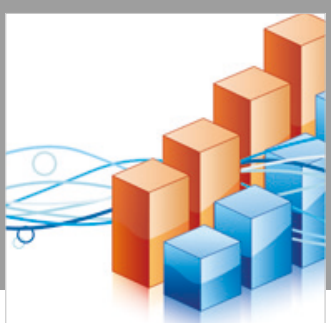

Advances in

Operations Research

\section{-n-m}
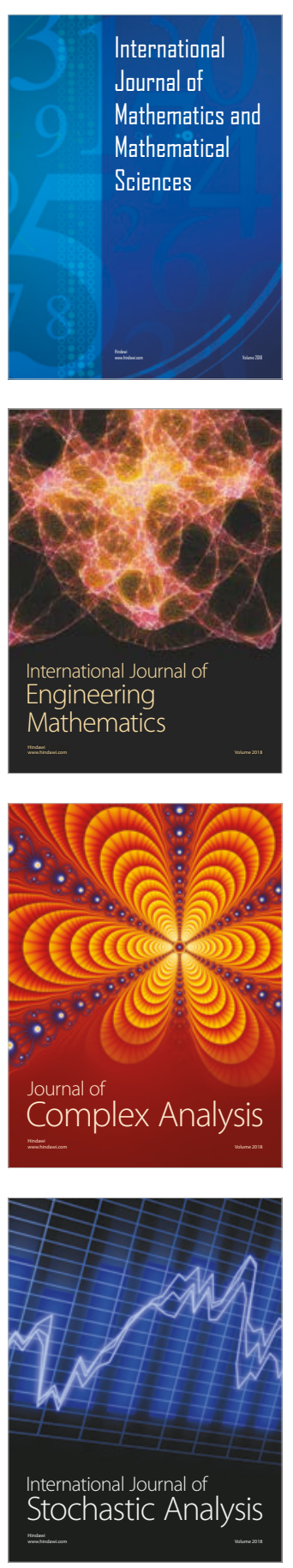
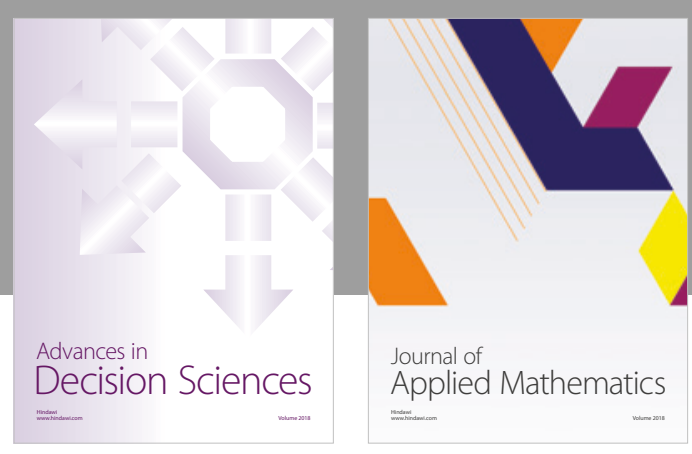

Journal of

Applied Mathematics
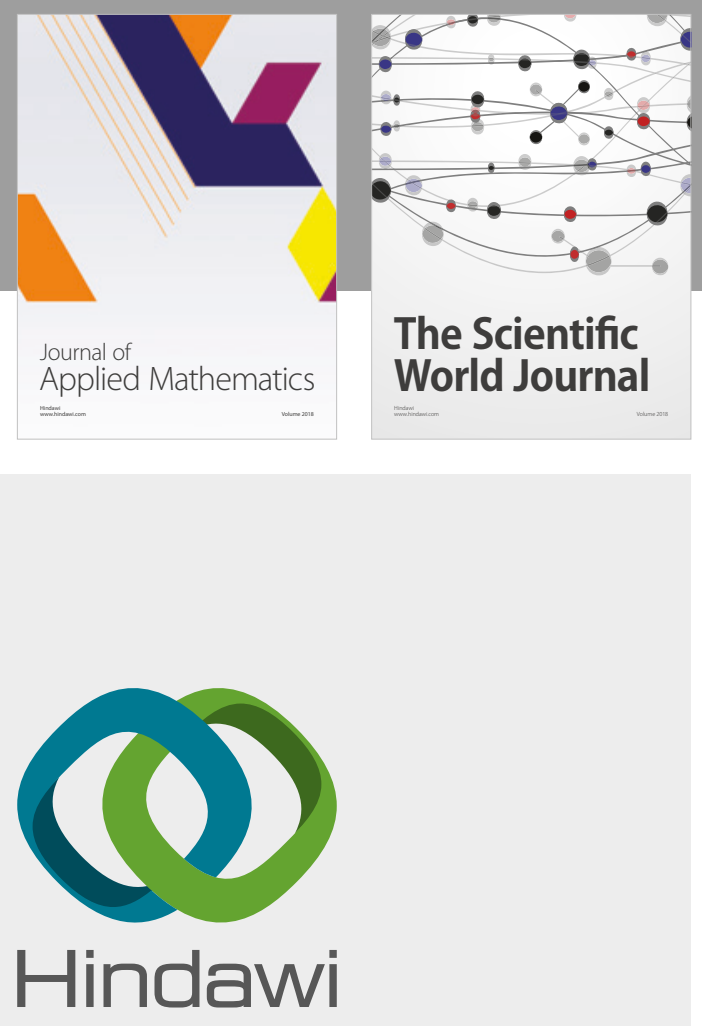

Submit your manuscripts at

www.hindawi.com

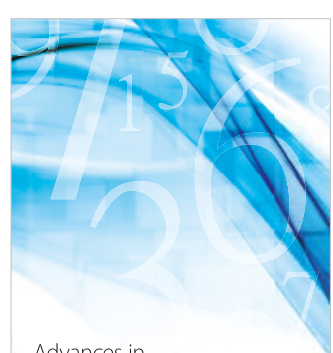

Advances in
Numerical Analysis
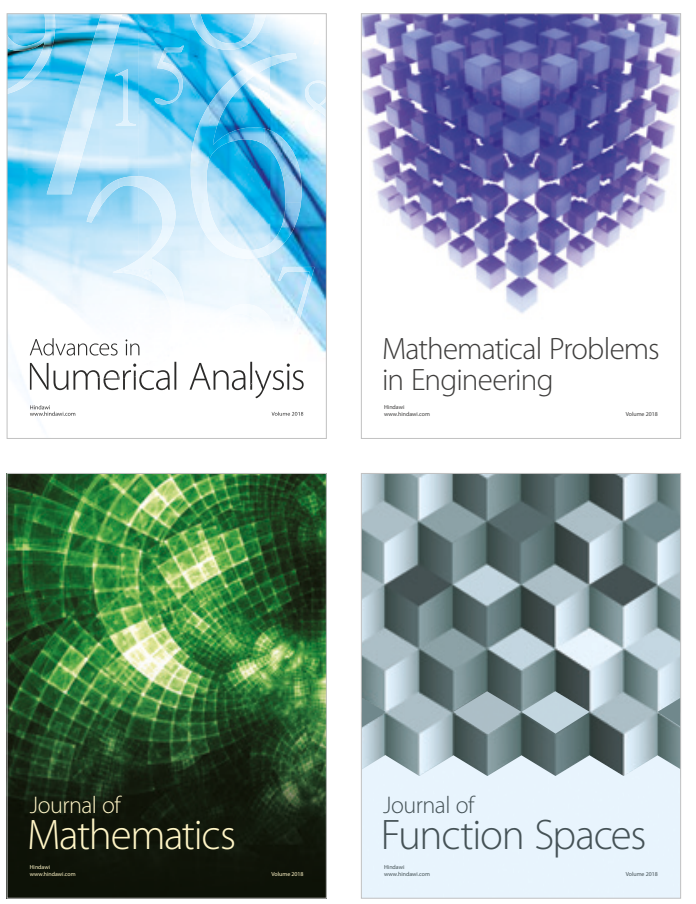

Mathematical Problems in Engineering

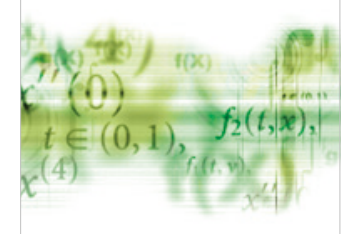

International Journal of

Differential Equations

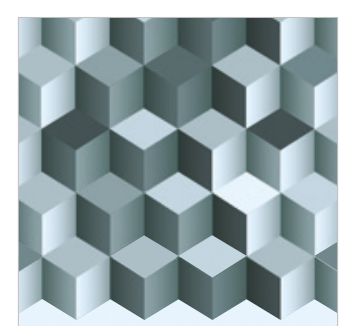

Journal of

Function Spaces
The Scientific

World Journal

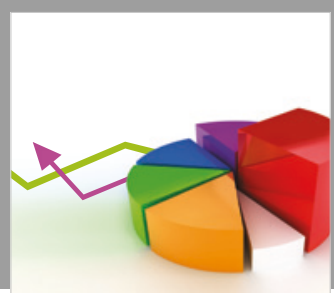

Journal of

Probability and Statistics
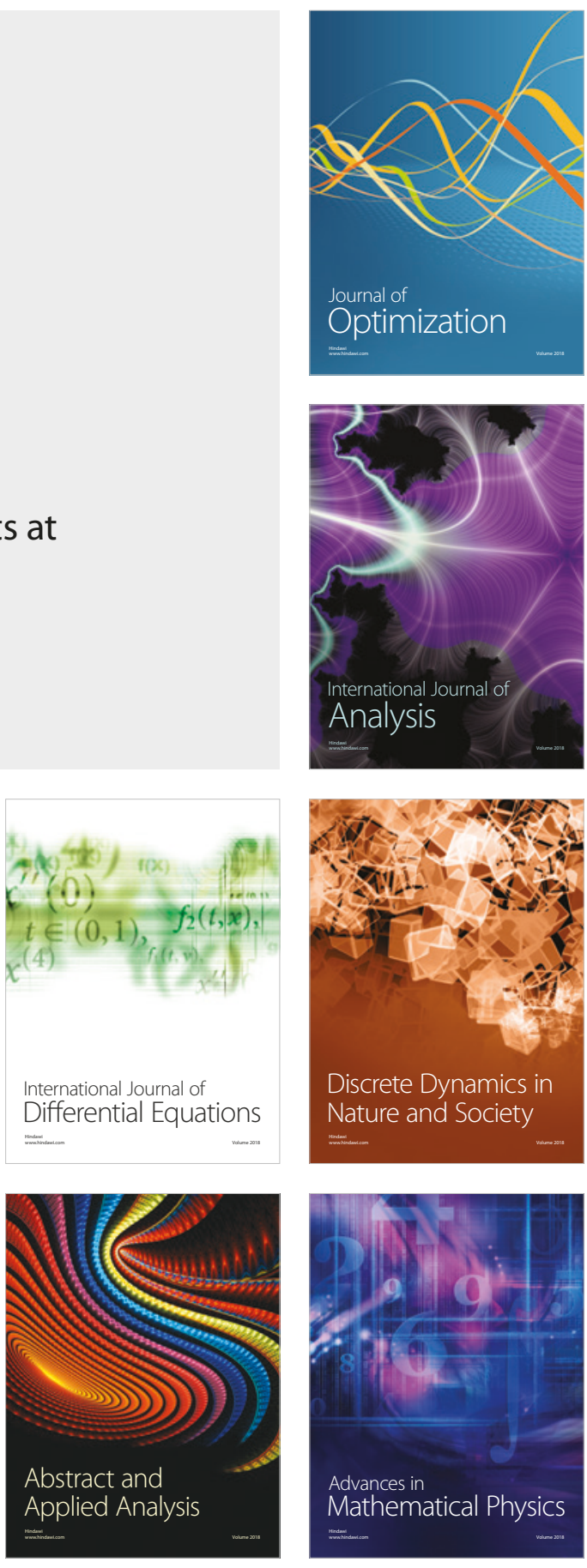